OPEN ACCESS

Edited by:

Hari S. Sharma,

Uppsala University, Sweden

Reviewed by:

J. Luis Lujan,

Mayo Clinic College of Medicine

and Science, United States

Vassiliy Tsytsarev,

University of Maryland, College Park,

United States

*Correspondence: Jose Gomez-Tames

jgomez@nitech.ac.jp

Specialty section:

This article was submitted to

Neural Technology,

a section of the journal

Frontiers in Neuroscience

Received: 23 March 2021 Accepted: 08 June 2021

Published: 30 June 2021

Citation:

Gomez-Tames J, Asai A and

Hirata A (2021) Multiscale

Computational Model Reveals Nerve

Response in a Mouse Model

for Temporal Interference Brain

Stimulation

Front. Neurosci. 15:684465 doi: 10.3389/fnins.2021.684465

\section{Multiscale Computational Model Reveals Nerve Response in a Mouse Model for Temporal Interference Brain Stimulation}

\author{
Jose Gomez-Tames ${ }^{1,2 *}$, Akihiro Asai' and Akimasa Hirata ${ }^{1,2}$ \\ ${ }^{1}$ Department of Electrical and Mechanical Engineering, Nagoya Institute of Technology, Nagoya, Japan, ${ }^{2}$ Center \\ of Biomedical Physics and Information Technology, Nagoya Institute of Technology, Nagoya, Japan
}

There has been a growing interest in the non-invasive stimulation of specific brain tissues, while reducing unintended stimulation in surrounding regions, for the medical treatment of brain disorders. Traditional methods for non-invasive brain stimulation, such as transcranial direct current stimulation (tDCS) or transcranial magnetic stimulation (TMS), can stimulate brain regions, but they also simultaneously stimulate the brain and non-brain regions that lie between the target and the stimulation site of the source. Temporal interference (TI) stimulation has been suggested to selectively stimulate brain regions by superposing two alternating currents with slightly different frequencies injected through electrodes attached to the scalp. Previous studies have reported promising results for $\mathrm{TI}$ applied to the motor area in mice, but the mechanisms are yet to be clarified. As computational techniques can help reveal different aspects of $\mathrm{TI}$, in this study, we computationally investigated TI stimulation using a multiscale model that computes the generated interference current pattern effects in a neural cortical model of a mouse head. The results indicated that the threshold increased with the carrier frequency and that the beat frequency did not influence the threshold. It was also found that the intensity ratio between the alternating currents changed the location of the responding nerve, which is in agreement with previous experiments. Moreover, particular characteristics of the envelope were investigated to predict the stimulation region intuitively. It was found that regions with high modulation depth (| maximum| - | minimum/ values of the envelope) and low minimum envelope (near zero) corresponded with the activation region obtained via neural computation.

Keywords: transcranial temporal interference stimulation, brain stimulation, multiscale model, mouse model, neural model, envelope

\section{INTRODUCTION}

There has been increasing interest in the non-invasive electrostimulation of specific parts of the brain. Recently, as one of the topics in this field, stimulation of the deep brain region has gained attention (DaSilva et al., 2015; Csifcsák et al., 2018; Gomez-Tames et al., 2019a, 2020a; Huang and Parra, 2019; Bikson and Dmochowski, 2020). In conventional non-invasive stimulation techniques, such as transcranial electrical stimulation [e.g., transcranial alternating current stimulation (tACS); 
transcranial direct current stimulation (tDCS)] and transcranial magnetic stimulation (TMS), can stimulate specific brain regions, but they also simultaneously stimulate the surrounding brain and non-brain regions (e.g., nociceptive fibers stimulation in the scalp) that lie between the stimulator location and target area (Chib et al., 2013; Weber et al., 2014; Huang et al., 2017; Bikson and Dmochowski, 2020; Gomez-Tames et al., 2020a, 2021).

Temporal interference (TI) stimulation has attracted significant attention as it may achieve stimulation of specific cortical or deep brain regions without activation of superficial parts (Laakso and Hirata, 2013; Vossen et al., 2015). TI makes use of two sets of tACS, whose injection current frequencies slightly differ from each other, that may cause a beat wave at a specific area of the brain, enabling position-selective stimulation (Grossman et al., 2017). This stimulation method has been conventionally applied to peripheral stimulation, such as sacral nerve stimulation (Johnson and Tabasam, 2003; Beatti et al., 2011). TI can be considered for stimulation due to the low-pass filtering effect of the passive cell membrane that may be accompanied by rectification of the ionic part (Middleton et al., 2006; Grossman et al., 2017; Mirzakhalili et al., 2020). If a current (a few $\mathrm{kHz}$ up to $10 \mathrm{kHz}$ ) is injected, the cell membrane in the brain may not readily follow the oscillation of the electric field. Instead, a small difference in the frequencies of the two injection currents may generate a beat wave (modulation envelope) causing neural stimulation. Previous studies have shown promising results for motor area stimulation in mice (Grossman et al., 2017; Song et al., 2020). In addition, various efforts have been made to clarify the mechanism regarding biophysics using neural models (Karimi et al., 2019; Cao and Grover, 2020; Howell and McIntyre, 2020; Mirzakhalili et al., 2020; Esmaeilpour et al., 2021). It is also relevant to incorporate and validate the effects of the interferential currents that are shaped by the anatomical and electrical properties of biological tissues (Rampersad et al., 2019).

In the non-invasive brain stimulation studies, the electric current, as a physical agent, is computed using a volume conductor model, in which the human head (not only the brain) is considered as an inhomogeneous conductor without considering the neural model (Datta et al., 2009; Bikson et al., 2013; Laakso et al., 2015; Opitz et al., 2015; Antonenko et al., 2019; Kasten et al., 2019). Here, the electric current is shaped by the electrical and anatomical characteristics of the head. However, the multiscale modeling, or the interaction with the axon, is needed to replicate the neuromodulation effects in the computational domain (Wongsarnpigoon and Grill, 2008; Salvador et al., 2011; Gomez-Tames et al., 2019b; Seo and Jun, 2019). Several studies have been conducted for multiscale modeling, particularly for TMS (De Geeter et al., 2015; Goodwin and Butson, 2015; Gomez-Tames et al., 2019c), where the pulse frequency is of the order of $\mathrm{kHz}$ that have been able to predict experimental thresholds (Gomez-Tames et al., 2018, 2020b; Aberra et al., 2020). If the stimulation of the mouse cortex can be explained in terms of multiscale modeling, the abovementioned hypothesis can be clarified, except for the possible synaptic effect (Gomez-Tames et al., 2019b; Neudorfer et al., 2021).
Here, we present multiscale computational modeling to explore the effects of interferential stimulation on the mouse motor cortex for first time based on experimental measurements. In addition, the characteristics of the generated envelope were explored to intuitively predict the stimulation region.

\section{MODEL AND METHODS}

\section{Mouse Model}

The mouse model used in this study was developed using computer tomography (Dogdas et al., 2007). The model has the resolution of $0.1 \mathrm{~mm}$ and comprises 21 tissues. The dimensions of the model are $38.0 \mathrm{~mm} \times 99.2 \mathrm{~mm} \times 20.8 \mathrm{~mm}$, excluding the tail. In this study, the model was truncated around the neck region because the remaining body part did not influence the current flow in the brain. The head model includes six tissues, namely, skin, brain, muscle, bone, eye, and glands, as shown in Figure $\mathbf{1}$. Owing to the limitation of image resolution, the thickness of the cerebrospinal fluid is not well modeled.

\section{Volume Conductor Model}

The electric potential generated by the current injected from the electrodes attached to the rat scalp was computed using the scalar potential finite-difference method, with successive overrelaxation and multigrid methods (Dawson and Stuchly, 1998; Laakso and Hirata, 2012) to solve the scalar potential equation:

$$
\nabla \cdot\left(\sigma \nabla V_{e}\right)=0,
$$

where $V_{e}$ and $\sigma$ denote the scalar potential and tissue conductivity, respectively. Then, the electric field was obtained by dividing the potential between the two nodes along the edge of a cubic voxel (the minimum component of the model) by the length of the voxel edge. We assigned the electrical conductivity of tissue to each tissue based on the fourth order Cole-Cole model at $1 \mathrm{kHz}$ and literature values (Gabriel et al., 1996; Bernabei et al., 2014). The electrical conductivity did not change significantly in the lower $\mathrm{kHz}$ range; thus, the value of $1 \mathrm{kHz}$ is used throughout this study. The electrical conductivities of the skin, brain, muscle, bone, eye, and glands were $0.1,0.33,0.321$, $0.02,1.5$, and $0.67 \mathrm{~S} / \mathrm{m}$, respectively.

\section{Axon Model}

The effects of the extracellular electric field on nerve axons were described by the following general equation (McNeal, 1976; Rattay, 1999):

$$
\begin{gathered}
c_{m, n} \frac{d V_{m, n}}{d t}+I_{i o n, n}-\frac{V_{m, n-1}-2 V_{m, n}+-2 V_{m, n+1}}{R} \\
=\frac{V_{e, n-1}-2 V_{e, n}+-2 V_{e, n+1}}{R},
\end{gathered}
$$

where $c_{m, n}$ is the membrane capacitance, and $V_{m, n}$ is the membrane potential at position $n$. The interferential current $V_{e, n}$ was obtained using the scalar potential finite-difference, and the axon of a myelinated nerve consists of internodes (segments 


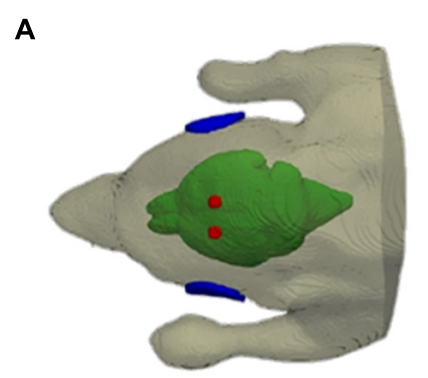

B

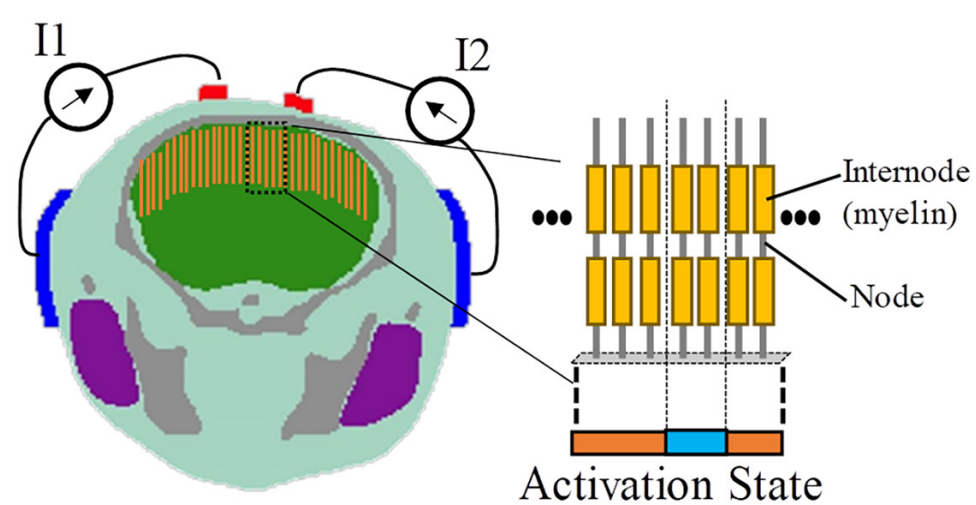

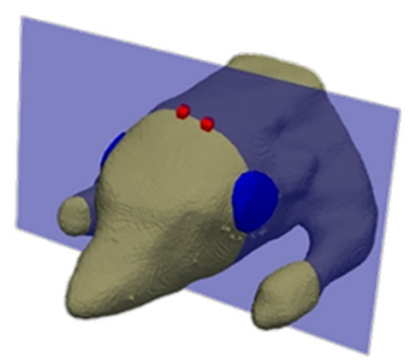

$\square$ Anode

$\square$ Brain

$\square$ Muscle

$\square$ Cathode

Skin

Bone

\section{$\square$ Nerve axons}

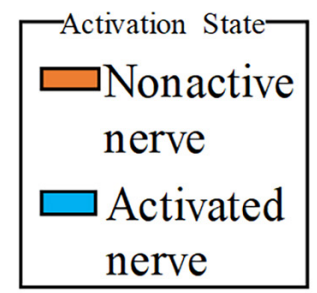

FIGURE 1 | Multiscale model for interferential transcranial alternate current stimulation. (A) Volume conductor of the mouse model with two pairs of electrodes attached on the scalp based on the experimental scenario in Grossman et al. (2017). (B) Cross-section showing the positions of the axonal nerves in the motor area of the brain (total number of test axons is 80 ). The activated nerves are depicted by an activation state region (1D).

ensheathed by myelin) and nodes of Ranvier (ionic channels). The variable $R=0.5 \times\left(R_{n}+R_{n+1}\right)$ denotes the intra-axonal resistance between the centers of two adjacent compartments (nodes and internodes). At the myelinated internodes, the leak conductance was modeled as a passive element. At the nodes of Ranvier, the ionic membrane current was formulated as a conductance-based voltage-gated model based on the ChiuRitchie-Rogart-Stagg-Sweeney model (Sweeney et al., 1987), which has been able to reproduce experimental results for motor stimulation of the brain cortex (Gomez-Tames et al., 2018, 2019b). The model parameters are presented in Table 1. The length and diameter of an axon in a mouse have been reported to be $2 \mathrm{~mm}$ and $1 \mu \mathrm{m}$, respectively (Ong et al., 2009). No measured results have been reported for the myelin thickness. Thus, the original parameters $C_{m, i n}$ and $C_{m, n}$ were increased linearly by a factor of 15, considering that the thickness of the myelin reduces with the ratio of axon thickness of a human to that of a mouse (Arancibia-Cárcamo et al., 2017). No adjustments were applied for fine-tuning. Due to the lack of detailed anatomy of the mouse brain, we simply located the axons perpendicular to the brain cortex, as shown in Figure 1.

Finally, the required injection current was obtained to propagate an action potential in each axon of Figure 1B using a search method (bisection method) until the error was smaller than $10 \mu \mathrm{A}$. An action potential was elicited when the membrane potential was depolarized up to $80 \mathrm{mV}$ in at
TABLE 1 | Parameters of the modified CRRSS model.

\begin{tabular}{ll}
\hline Parameter & Value \\
\hline Nernst potential for sodium channels $\left(V_{\mathrm{Na}}\right)$ & $115 \mathrm{mV}$ \\
Nernst potential for leakage channels $\left(V_{l}\right)$ & $-0.01 \mathrm{mV}$ \\
Capacity of membrane at internode $\left(C_{m}\right)$ & $402 \mathrm{nF}$ \\
Capacity of membrane at node $\left(C_{m}\right)$ & $452 \mathrm{nF}$ \\
Internode membrane resistivity $\left(R_{m}\right)$ & $219 \mathrm{k} \Omega$ \\
Nodal membrane resistivity $\left(R_{m}\right)$ & $3.26 \mathrm{k} \Omega$ \\
Myeline conductance $\left(G_{m}\right)$ & $26.8 \mathrm{nS}$ \\
Sodium channel conductance $\left(G_{n a}\right)$ & $1.445 \mathrm{kS}$ \\
Leaked channel conductance $\left(G_{l}\right)$ & $128 \mathrm{~S}$ \\
\hline
\end{tabular}

least four neighboring nodes at successive times (Reilly, 2016). Then, the computational activation threshold of TI was the minimum required injection current among all the test axons. The total number of test axons was 80 . This corresponds to a separation distance from each other of $0.1 \mathrm{~mm}$ (same to the mouse head model resolution). The axon with $0.1-\mathrm{mm}$ resolution was sufficient to determine the "activation state region" (see Figure 1B). This is based on the assumption that the field distribution is rather smooth, and thus if a higher number of axons were considered, the "activation state region" does not change as the additional axons between the activated axons would be stimulated simultaneously and vice versa. 

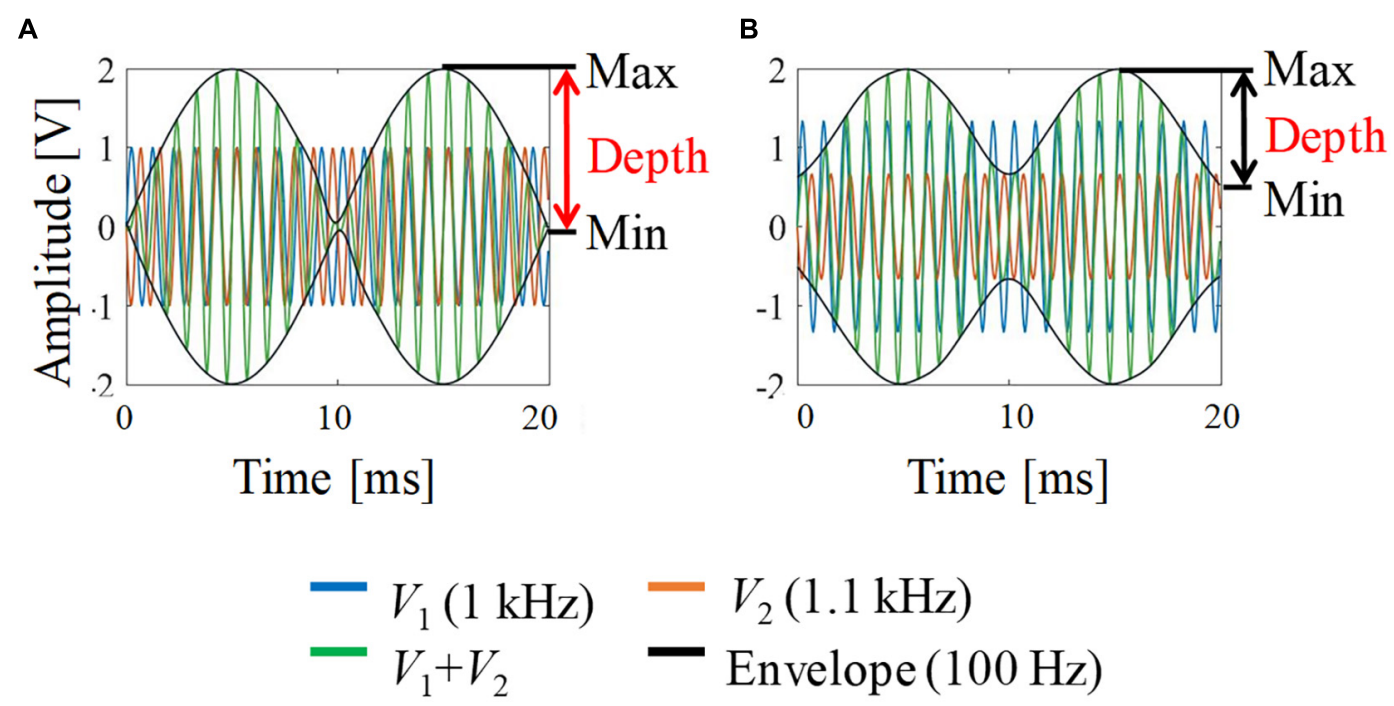

FIGURE 2 | Envelope modulation waveforms. (A) $V_{1}=V_{2} ; \mathbf{( B )} V_{1}: V_{2}=2: 1$. For illustration, the carrier frequency was chosen as $1 \mathrm{kHz}$ and the frequency difference (beat frequency) was $100 \mathrm{~Hz}$ (i.e., 1 and $1.1 \mathrm{kHz}$ ).

\section{Stimulation Scenarios}

The stimulation condition is shown in Figure 1, which was defined following the mouse experiment in Grossman et al. (2017). To replicate the scenario in Grossman et al. (2017), the diameters of the circular anode and cathode were chosen as 1 and $5 \mathrm{~mm}$, respectively, with a thickness of $0.5 \mathrm{~mm}$. In addition, the electrodes comprised a sponge with saline solution. Two tACS circuits were modeled. Each anode was attached around the top of the brain, with its respective cathode attached to the side of the head (injection currents in the right and left hemispheres are defined as $I_{1}$ and $I_{2}$, respectively).

In this study, we fixed the total current injected into the two electrodes as $0.776 \mathrm{~mA}\left(I_{1}+I_{2}\right)$, which is the mean value reported in Grossman et al. (2017). The carrier frequencies of tACS were chosen as $1,2,3$, and $4 \mathrm{kHz}$, and their differences were 5, 10, 15, 20, 50, and $100 \mathrm{~Hz}$, as in Grossman et al. (2017).

Figure 2 shows a schematic explanation and definition of typical envelop-modulated waveforms. It indicates the maximum, minimum, and depth of the envelope, which were used to quantify the neural activation in this study.

\section{RESULTS}

\section{Verification of Multiscale Model}

The effect of the frequency difference (beat frequency) and carrier frequency of the two injection currents on the stimulation was evaluated computationally and compared with the motor threshold reported in a previous study (Grossman et al., 2017). The experimental motor threshold corresponded to activation of the right forepaw; thus, the target area was set to the expected motor area in the left hemisphere, where the thick nerves (corresponding to the pyramidal axon) were located (Neafsey et al., 1986). The computational activation threshold corresponded to the scenario using an amplitude ratio of $I_{1}$ and $I_{2}$ as the experiment.

As shown in Figure 3A, the beat frequency does not influence the activation threshold from 5 to $100 \mathrm{~Hz}$, which is in good agreement with the measured threshold (Grossman et al., 2017). This tendency was computationally the same up to $100 \mathrm{~Hz}$, although no measured results were reported above $15 \mathrm{~Hz}$. From Figure 3B, the threshold of the stimulation increased with the frequency from 1 to $4 \mathrm{kHz}$, which is also in good agreement with the measured values.

\section{Effect of Amplitude Ratio on Stimulation Region}

The effect of the amplitude ratio of the two injection currents on the stimulation was evaluated. Figure 4 shows the electric field direction (current direction) on the brain for different injection current ratios. The carrier and beat frequencies were $1 \mathrm{kHz}$ and $10 \mathrm{~Hz}$, respectively. As shown in this figure, the envelope depth of the electric field magnitude shifted in the direction opposite to that of the electrode, whose injection current amplitude was higher. Similarly, the multiscale model demonstrates that the activated neurons varied according to the amplitude ratios of the injection current, in which the activation changed in the same manner as the envelope depth. These results agree with the experimental results (Grossman et al., 2017).

\section{Metrics for Neural Stimulation Estimation}

The different characteristics of the generated modulated signals of the electric field and electric potential were explored. Specifically, estimations of neuronal stimulation related to the maximum, minimum, and depth of the envelope were evaluated (see Figure 2). 


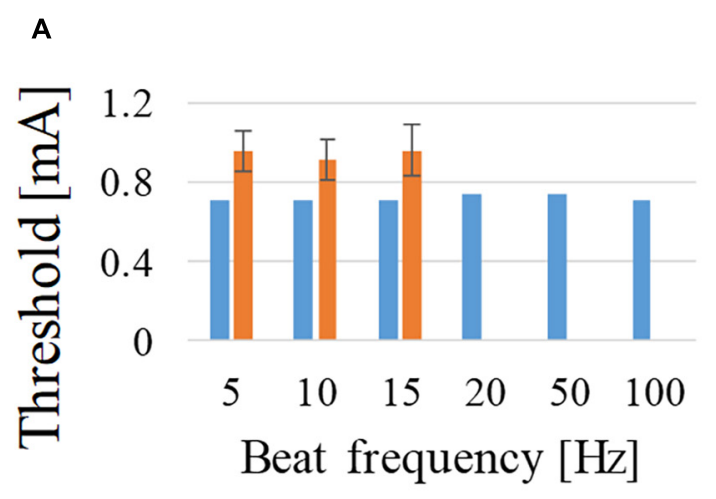

Simulation

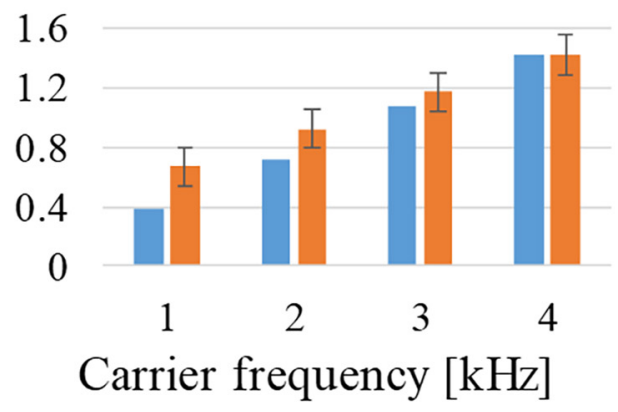

Experiment

FIGURE 3 | Effect on the activation threshold of $\mathbf{( A )}$ beat frequency at constant carrier frequency $(2 \mathrm{kHz})$ and $\mathbf{( B )}$ carrier frequency at constant beat frequency (10 Hz) using amplitude ratio of $I_{1}$ and $l_{2}$ of 7:3. For comparison, experimental observations of behavioral stimulation are also shown.

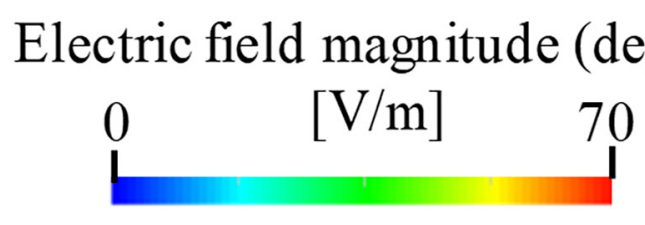

\section{$2: 8$}

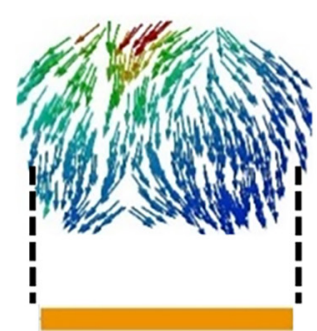

$3: 7$
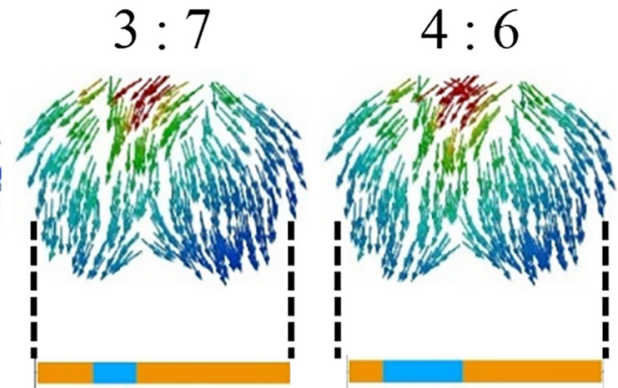

$4: 6$

$\left(I_{1}: I_{2}\right)$

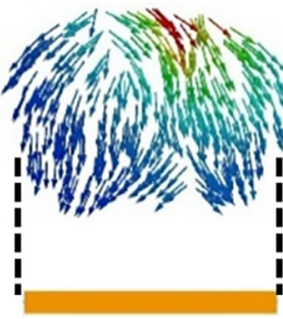

$8: 2$

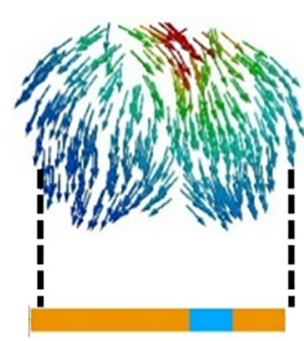

$7: 3$

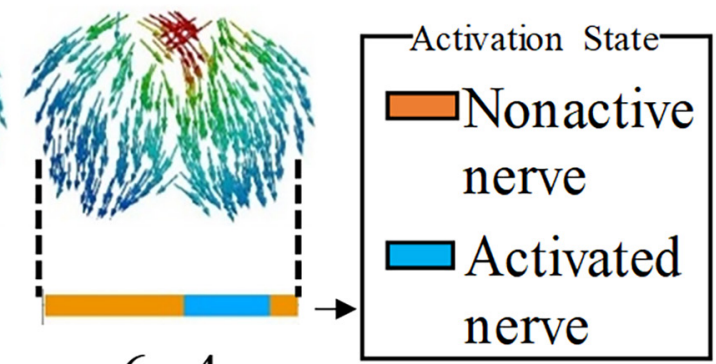

$6: 4$

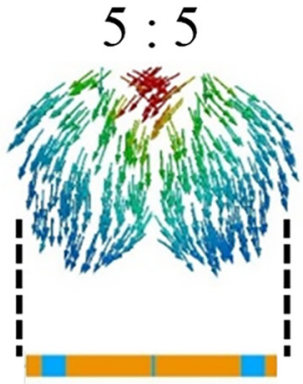

FIGURE 4 | Stimulation regions of different stimulation conditions $\left(I_{1}: /_{2}\right)$. The electric field vector direction was modified according to the injection current ratio (transverse plane). The magnitude is given by the envelope depth (see definition in Figure 2). Effects on neural activation are shown by the activation region that represents the state of the axon (active or non-active) for the different injection current ratios.

Figures 5, 6 show the electric potential and electric field distributions, respectively. We observed that the region of the maximum value of the envelope (electric potential and electric field) cannot predict the region of the activated fibers. In contrast, the hotspot of the envelope depth agrees with the activated nerve region estimated using the multiscale model. In addition, the 




FIGURE $\mathbf{5}$ | Electric potential distribution of the envelope for three metrics: maximum, minimum, and depth (see definitions in Figure 2). Activation state of the axons
are indicated.

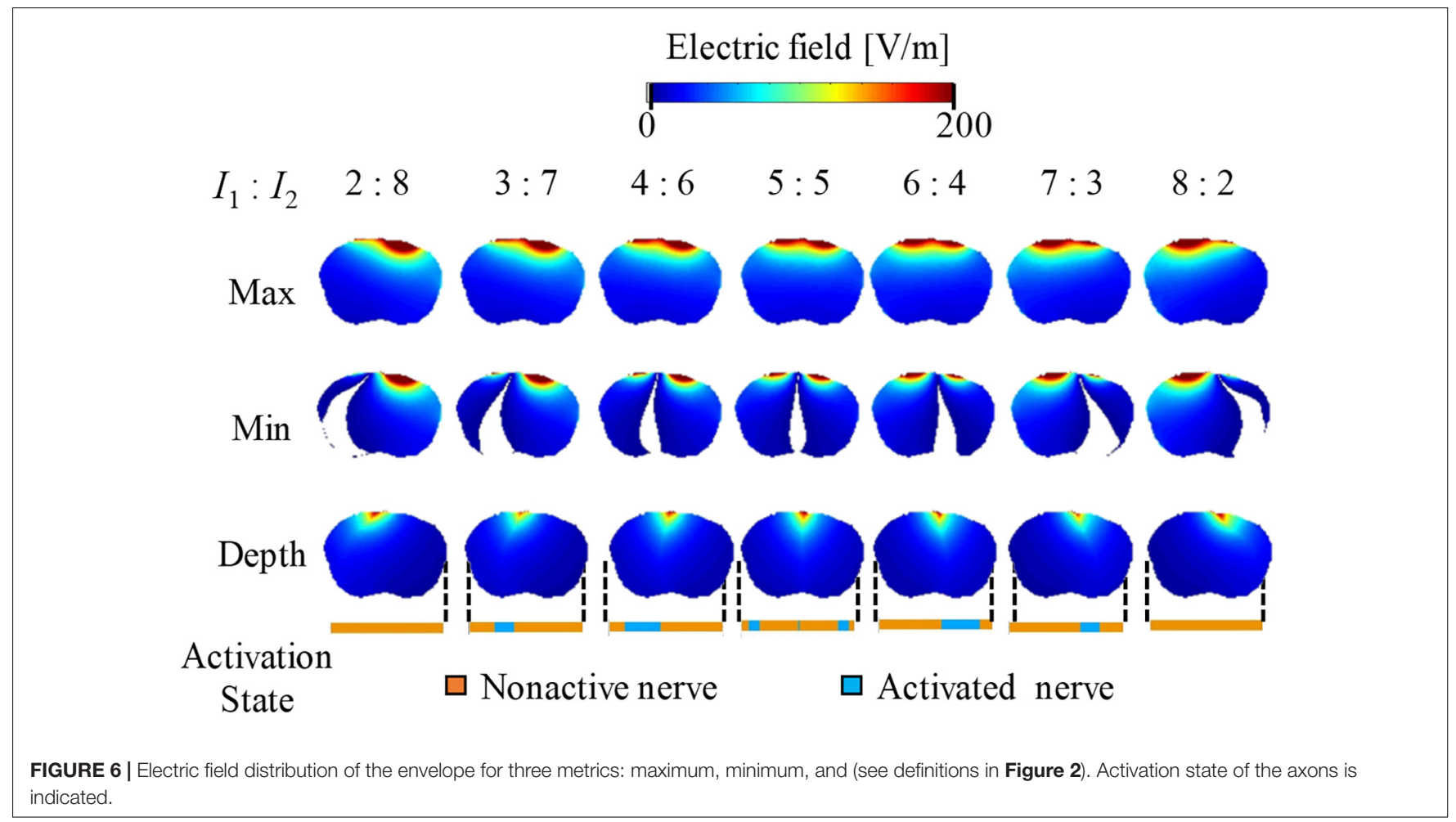

minimum value of the envelope (near zero) can be used to estimate the activated nerve region. In the case of depth, we noticed that the position of the hotspot using the electric potential envelope agreed with the center of the activated nerve region.
Figure 7 presents the relationship between the depth and minimum values of the envelope with the activated and non-activated nerves (case 7:3) based on multiscale model computations. In addition, the regions were separated into three 

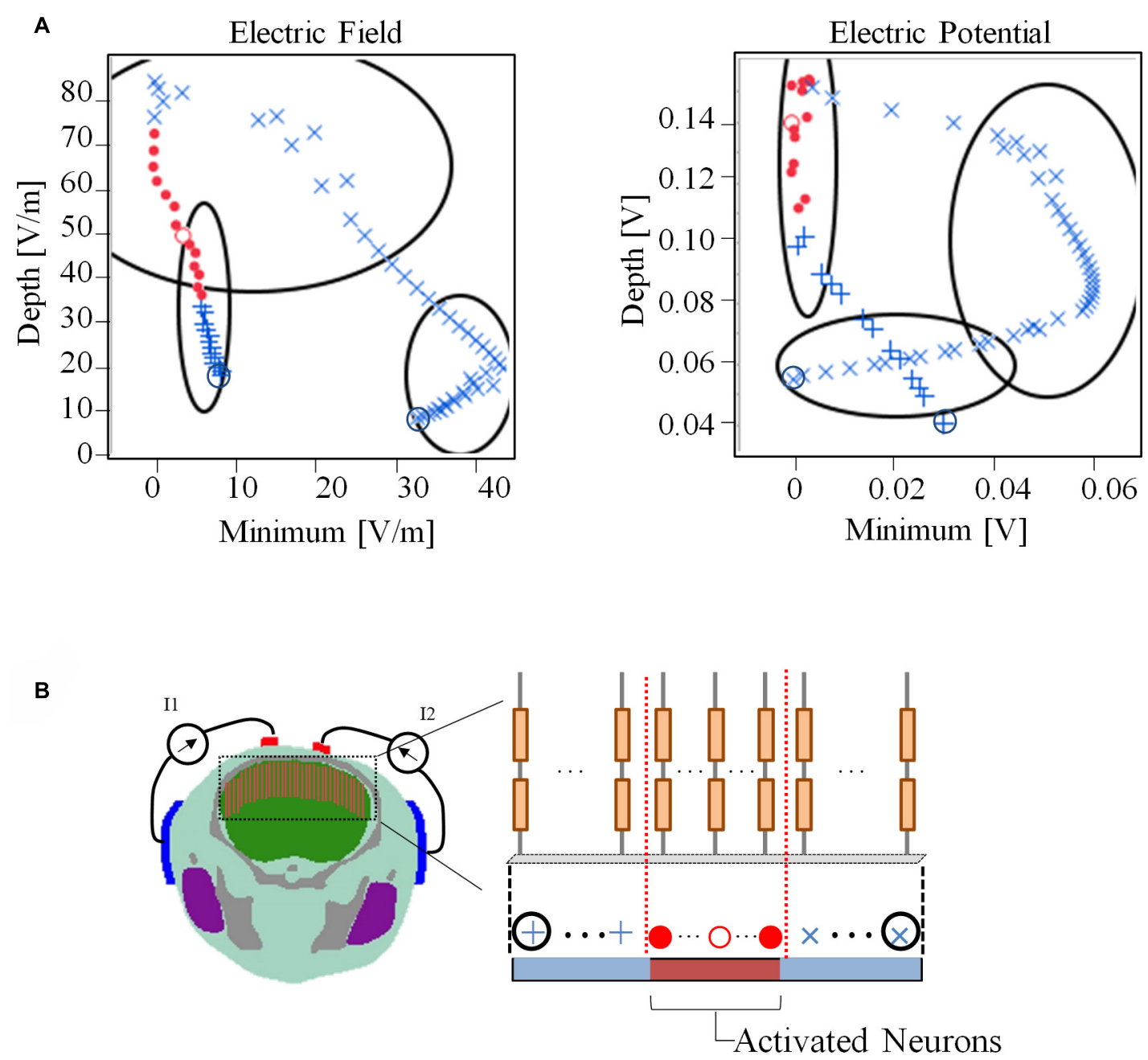

FIGURE 7 | (A) Relationship between depth and minimum of the envelope for detection of activated neurons using electric field and electric potential. The regions have been separated into three groups based only on the depth and minimum values using hierarchical clustering. (B) The activation state (active or non-active) is coded in red and blue colors based on the multiscale model results. Consecutive neighboring nerve positions follow the path (+, , $\times$ ). The values of the electric field and electric potential are the averages along the nerve.

groups based on hierarchical clustering. The computed activation region can be estimated based on the depth and minimum values of the electric potential envelope. Only considering the depth metric may determine the stimulation with the cost of including some non-active neurons next to the activation region. Whereas, only considering the minimum may include non-active neurons far from the activation region. Based on depth and minimum values of the electric field, the estimation group included more non-active neurons and more limited in the prediction.

\section{DISCUSSION}

In this study, we computationally investigated the effects of interference current patterns in a neural model embedded in a mouse brain using a multiscale model approach. To replicate the neuromodulation effects in the computational domain, we considered neural stimulation generated by interferential electric currents shaped by electrical and anatomical properties of the tissues in a realistic mouse head model for the first time. The model reproduced the experimental results by showing that that interferential stimulation acts on the neuronal axons. Also, this study showed which characteristics of the generated envelope can be used to predict the stimulation region to facilitate computational analysis intuitively.

Multiscale modeling was applied to replicate the experiments conducted in Grossman et al. (2017), the primary findings of which were: (i) beat frequency does not influence the threshold for the range considered, (ii) the threshold increases with an increase in the carrier frequency, and (iii) the stimulation region changes according to the ratio between the two injection currents. These were also reproduced in another study (Song et al., 2020). Our multiscale modeling replicated the three experiments for the first time using realistically shaped generated current effects 
on the neuronal model. Regarding the beat frequency effect, it is possible that the thresholds of the motor cortex may change at higher beat rates considering tetanic contraction of the muscle response. A larger current at a higher carrier frequency is necessary because a higher current amplitude is needed to overcome the low-pass filtering effect of the cell membrane. Furthermore, we computationally confirmed that the stimulated area can be controlled using the injected currents from two pairs of electrodes, which is one of the more important features of interferential stimulation to be exploited in brain stimulation techniques. Some differences between the computed and measured thresholds, as in Figure 3, are attributable to the positioning error in the electrode and/or fair modeling of the mouse brain (particularly for the cerebrospinal fluid), where magnetic resonance images may have insufficient resolution. Consequently, the nerve location was empirical.

We then explored the features of the generated envelope signal on the brain to determine which characteristics may serve as a physical metric of stimulation based on validated multiscale modeling. First, we observed that the maximum value of the generated electric field or electric potential envelope did not correspond to the exact activated nerve region given by the multiscale model. Instead, high values of the "depth" parameter (| maximum $|-|$ minimum| values of the envelope) corresponded to better predictions of the activation region. We also noticed that the minimum value near zero (100\% modulation) of the envelope may be used to determine the activated nerve region, which is the region in which both injection currents have the same intensity. Moreover, the combination of these metrics may help to better characterize the activated nerve region predicted using the multiscale model.

A limitation of our computational model is summarized as follows: first, the mouse model did not consider the cerebrospinal fluid, whose conductivity is higher than those of the remaining tissues, altering the current direction. However, the medical images, in general, do not warrant a resolution of less than $0.1 \mathrm{~mm}$, and thus, unlike humans, tissue segmentation is insufficient. Therefore, the current flow due to the complexity of the brain might not be well represented. Also, neural trajectories have a radial orientation of neuronal paths from the cortex in the mouse (Wang et al., 2020). Our assumption of perpendicular axons for the sake of simplicity is more suitable for flat somatomotor cortical areas near the central part. Also, the effect of the axon curvature in the interior has a negligible effect as the stimulation occurs on the upper parts of the neuron (Gomez-Tames et al., 2020b).

In terms of validation where the experimental data is available (Grossman et al., 2017), the motor cortex was chosen. This area

\section{REFERENCES}

Aberra, A. S., Wang, B., Grill, W. M., and Peterchev, A. V. (2020). Simulation of transcranial magnetic stimulation in head model with morphologicallyrealistic cortical neurons. Brain Stimul. 13, 175-189. doi: 10.1016/j.brs.2019.1 0.002 is suitable as it provides an easy marker of the stimulation effect to understand TI stimulation. Our findings can be extended to different brain areas, where the stimulation is characterized by axon stimulation. Also, the envelope formation at a specific part is expected to be useful to other brain regions. In addition, the cortical area is also a relevant target to investigate TI selectivity in the next study. For instance, generated scalp pain limits the intensity of injection currents in transcranial electrical stimulation (up to a few $\mathrm{mA}$ ), producing weak electric fields in the brain. Instead, TI stimulation can selectively stimulate specific cortical parts while avoiding co-stimulation of peripheral scalp nerves. The next steps need to consider adding neural models not only in the cortical region but also in deep brain regions and peripheral nerves on the scalp (e.g., pain perception) to evaluate the reduction of co-stimulation.

In conclusion, we examined the effects of interferential stimulation on a neural model of the motor cortex. We considered the effects of the injection current on a realistic mouse head model. The results confirmed that varying the carrier frequency does not affect the threshold value, and the threshold value increases with an increase in the carrier frequency. The verified multiscale model for interferential stimulation was used to reveal the significant characteristics of the envelope generated by interferential stimulation to facilitate the estimation of the stimulation region. In particular, regions with a high modulation depth and low minimum envelope correspond to the activation region.

\section{DATA AVAILABILITY STATEMENT}

The raw data supporting the conclusions of this article will be made available by the authors, without undue reservation.

\section{AUTHOR CONTRIBUTIONS}

AH and JG-T conceived and designed the study. JG-T developed the interferential multiscale model. AA conducted the simulation experiments. AA and JG-T processed the data. All authors analyzed the data, wrote the manuscript, and read and approved the manuscript.

\section{FUNDING}

This study was supported by a JSPS Grant-in-Aid for Scientific Research, JSPS KAKENHI Grant-17H00869, 18H03133, 19K20668, and 21H04956.

Antonenko, D., Thielscher, A., Saturnino, G. B., Aydin, S., Ittermann, B. Grittner, U., et al. (2019). Towards precise brain stimulation: is electric field simulation related to neuromodulation? Brain Stimul. 12, 1159-1168. doi: 10.1016/J.BRS.2019.03.072

Arancibia-Cárcamo, I. L., Ford, M. C., Cossell, L., Ishida, K., Tohyama, K., and Attwell, D. (2017). Node of ranvier length as a potential regulator 
of myelinated axon conduction speed. Elife 6:e23329. doi: 10.7554/eLife.2 3329

Beatti, A., Rayner, A., Chipchase, L., and Souvlis, T. (2011). Penetration and spread of interferential current in cutaneous, subcutaneous and muscle tissues. Physiotherapy 97, 319-326. doi: 10.1016/j.physio.2011.01.008

Bernabei, J. M., Lee, W. H., and Peterchev, A. V. (2014). "Modeling transcranial electric stimulation in mouse: a high resolution finite element study", in Proceedings of the 2014 36th Annual International Conference of the IEEE Engineering in Medicine and Biology Society. EMBC, Vol 2014, Chicago, IL, 406-409. doi: 10.1109/EMBC.2014.6943614

Bikson, M., and Dmochowski, J. (2020). What It means to go deep with noninvasive brain stimulation. Clin. Neurophysiol. 131, 752-754. doi: 10.1016/j. clinph.2019.12.003

Bikson, M., Dmochowski, J., and Rahman, A. (2013). The 'quasi-uniform' assumption in animal and computational models of non-invasive electrical stimulation. Brain Stimul. 6, 704-705. doi: 10.1016/j.brs.2012.11.005

Cao, J., and Grover, P. (2020). STIMULUS: noninvasive dynamic patterns of neurostimulation using spatio-temporal interference. IEEE Trans. Biomed. Eng. 67, 726-737. doi: 10.1109/TBME.2019.2919912

Chib, V. S., Yun, K., Takahashi, H., and Shimojo, S. (2013). Noninvasive remote activation of the ventral midbrain by transcranial direct current stimulation of prefrontal cortex. Transl. Psychiatry 3:e268. doi: 10.1038/tp.2013.44

Csifcsák, G., Boayue, N. M., Puonti, O., Thielscher, A., and Mittner, M. (2018). Effects of transcranial direct current stimulation for treating depression: a modeling study. J. Affect. Disord. 234, 164-173. doi: 10.1016/j.jad.2018.02.077

DaSilva, A. F., Truong, D. Q., DosSantos, M. F., Toback, R. L., Datta, A., and Bikson, M. (2015). State-of-art neuroanatomical target analysis of highdefinition and conventional TDCS montages used for migraine and pain control. Front. Neuroanat. 9:89. doi: 10.3389/fnana.2015.00089

Datta, A., Bansal, V., Diaz, J., Patel, J., Reato, D., and Bikson, M. (2009). Gyriprecise head model of transcranial direct current stimulation: improved spatial focality using a ring electrode versus conventional rectangular pad. Brain Stimul. 2, 201-207, 207.e1. doi: 10.1016/j.brs.2009.03.005

Dawson, T. W., and Stuchly, M. A. (1998). High-resolution organ dosimetry for human exposure to low-frequency magnetic fields. IEEE Trans. Magn. 34, 708-718. doi: $10.1109 / 20.668071$

De Geeter, N., Crevecoeur, G., Leemans, A., and Dupré, L. (2015). Effective electric fields along realistic DTI-based neural trajectories for modelling the stimulation mechanisms of TMS. Phys. Med. Biol. 60, 453-471. doi: 10.1088/0031-9155/60/ $2 / 453$

Dogdas, B., Stout, D., Chatziioannou, A. F., and Leahy, R. M. (2007). Digimouse: a 3D whole body mouse atlas from CT and cryosection data. Phys. Med. Biol. 52, 577-587. doi: 10.1088/0031-9155/52/3/003

Esmaeilpour, Z., Kronberg, G., Reato, D., Parra, L. C., and Bikson, M. (2021). Temporal interference stimulation targets deep brain regions by modulating neural oscillations. Brain Stimul. 14, 55-65. doi: 10.1016/j.brs.2020.11.007

Gabriel, S., Lau, R. W., and Gabriel, C. (1996). The dielectric properties of biological tissues: III. Parametric models for the dielectric spectrum of tissues. Phys. Med. Biol. 41, 2271-2293. doi: 10.1088/0031-9155/41/11/003

Gomez-Tames, J., Asai, A., and Hirata, A. (2020a). Significant group-level hotspots found in deep brain regions during TDCS: a computational analysis of electric field. Clin. Neurophysiol. 3, 755-765. doi: 10.1016/j.clinph.2019.11.018

Gomez-Tames, J., Asai, A., Mikkonen, M., Laakso, I., Tanaka, S., Uehara, S., et al. (2019a). Group-level and functional-region analysis of electric-field shape during cerebellar transcranial direct current stimulation with different electrode montages. J. Neural Eng. 16:036001. doi: 10.1088/1741-2552/ab0ac5

Gomez-Tames, J., Hirata, A., Tamura, M., and Muragaki, Y. (2019b). Corticomotoneuronal model for intraoperative neurophysiological monitoring during direct brain stimulation. Int. J. Neural Syst. 29:1850026. doi: 10.1142/S0129065718500260

Gomez-Tames, J., Kutsuna, T., Tamura, M., Muragaki, Y., and Hirata, A. (2018). Intraoperative direct subcortical stimulation: comparison of monopolar and bipolar stimulation. Phys. Med. Biol. 63:225013. doi: 10.1088/1361-6560/aaea06

Gomez-Tames, J., Laakso, I., Murakami, T., Ugawa, Y., and Hirata, A. (2020b). TMS activation site estimation using multiscale realistic head models. Iopscience.Iop.Org. 17:036004. doi: 10.1088/1741-2552/ab $8 \mathrm{ccf}$
Gomez-Tames, J., Tani, K., Hayashi, K., Tanaka, S., Ueno, S., and Hirata, A. (2021). Dosimetry analysis in non-brain tissues during TMS exposure of Broca's and M1 areas. Front. Neurosci. 15:154. doi: 10.3389/fnins.2021.644951

Gomez-Tames, J., Tarnaud, T., Miwa, K., Hirata, A., Van de Steene, T., Martens, L., et al. (2019c). Brain cortical stimulation thresholds to different magnetic field sources exposures at intermediate frequencies. IEEE Trans. Electromagn. Compat. 61, 1944-1952. doi: 10.1109/TEMC.2019.2943138

Goodwin, B. D., and Butson, C. R. (2015). Subject-specific multiscale modeling to investigate effects of transcranial magnetic stimulation. Neuromodulation 18 , 694-704. doi: 10.1111/ner.12296

Grossman, N., Bono, D., Dedic, N., Kodandaramaiah, S. B., Rudenko, A., Suk, H. J., et al. (2017). Noninvasive deep brain stimulation via temporally interfering electric fields. Cell 169, 1029-1041.e16. doi: 10.1016/j.cell.2017.05.024

Howell, B., and McIntyre, C. C. (2020). Feasibility of interferential and pulsed transcranial electrical stimulation for neuromodulation at the human scale. Neuromodulation. doi: 10.1111/ner.13137 [Epub ahead of print].

Huang, Y., Liu, A. A., Lafon, B., Friedman, D., Dayan, M., Wang, X., et al. (2017). Measurements and models of electric fields in the in vivo human brain during transcranial electric stimulation. Elife 6:e18834. doi: 10.7554/eLife.18834

Huang, Y., and Parra, L. C. (2019). Can transcranial electric stimulation with multiple electrodes reach deep targets? Brain Stimul. 12, 30-40. doi: 10.1016/ J.BRS.2018.09.010

Johnson, M. I., and Tabasam, G. (2003). An investigation into the analgesic effects of interferential currents and transcutaneous electrical nerve stimulation on experimentally induced ischemic pain in otherwise pain-free volunteers. Phys. Ther. 83, 208-223. doi: 10.1093/ptj/83.3.208

Karimi, F., Attarpour, A., Amirfattahi, R., and Nezhad, A. Z. (2019). Computational analysis of non-invasive deep brain stimulation based on interfering electric fields. Phys. Med. Biol. 64:235010. doi: 10.1088/1361-6560/ab5229

Kasten, F. H., Duecker, K., Maack, M. C., Meiser, A., and Herrmann, C. S. (2019). Integrating electric field modeling and neuroimaging to explain inter-individual variability of TACS Effects. Nat. Commun. 10:5427. doi: 10.1038/s41467-01913417-6

Laakso, I., and Hirata, A. (2012). Fast multigrid-based computation of the induced electric field for transcranial magnetic stimulation. Phys. Med. Biol. 57, 77537765. doi: 10.1088/0031-9155/57/23/7753

Laakso, I., and Hirata, A. (2013). Computational analysis shows why transcranial alternating current stimulation induces retinal phosphenes. J. Neural Eng. 10:46009. doi: 10.1088/1741-2560/10/4/046009

Laakso, I., Tanaka, S., Koyama, S., De Santis, V., and Hirata, A. (2015). Inter-subject variability in electric fields of motor cortical TDCS. Brain Stimul. 8, 906-913. doi: 10.1016/j.brs.2015.05.002

McNeal, D. R. (1976). Analysis of a model for excitation of myelinated nerve. IEEE Trans. Biomed. Eng. 4, 329-337. doi: 10.1109/tbme.1976.324593

Middleton, J. W., Longtin, A., Benda, J., and Maler, L. (2006). The cellular basis for parallel neural transmission of a high-frequency stimulus and its low-frequency envelope. Proc. Natl. Acad. Sci. U.S.A. 103, 14596-14601. doi: 10.1073/pnas. 0604103103

Mirzakhalili, E., Barra, B., Capogrosso, M., and Lempka, S. F. (2020). Biophysics of temporal interference stimulation. Cell Syst. 11, 557-572.e5. doi: 10.1016/j.cels. 2020.10.004

Neafsey, E. J., Bold, E. L., Haas, G., Hurley-Gius, K. M., Quirk, G., Sievert, C. F., et al. (1986). The organization of the rat motor cortex: a microstimulation mapping study. Brain Res. Rev. 11, 77-96. doi: 10.1016/0165-0173(86)90011-1

Neudorfer, C., Chow, C. T., Boutet, A., Loh, A., Germann, J., Elias, G. J. B., et al. (2021). Kilohertz-frequency stimulation of the nervous system: a review of underlying mechanisms. Brain Stimul. 14, 513-530. doi: 10.1016/j.brs.2021.03. 008

Ong, H. H., Wright, A. C., Wehrli, S. L., Souza, A., Schwartz, E. D., Hwang, N., et al. (2009). Indirect measurement of regional axon diameter in excised mouse spinal cord with Q-space imaging: simulation and experimental studies. Neuroimage 40, 1619-1632. doi: 10.1016/j.neuroimage.2008.01.017.Indirect

Opitz, A., Paulus, W., Will, S., Antunes, A., and Thielscher, A. (2015). Determinants of the electric field during transcranial direct current stimulation. NeuroImage 109, 140-150. doi: 10.1016/j.neuroimage.2015.01.033

Rampersad, S., Roig-Solvas, B., Yarossi, M., Kulkarni, P. P., Santarnecchi, E., Dorval, A. D., et al. (2019). Prospects for transcranial temporal interference 
stimulation in humans: a computational study. NeuroImage 202:116124. doi: 10.1016/j.neuroimage.2019.116124

Rattay, F. (1999). The basic mechanism for the electrical stimulation of the nervous system. Neuroscience 89, 335-346. doi: 10.1016/s0306-4522(98)00330-3

Reilly, P. (2016). Survey of numerical electrostimulation models. Phys. Med. Biol. 61, 4346-4363. doi: 10.1088/0031-9155/61/12/4346

Salvador, R., Silva, S., Basser, P. J., and Miranda, P. C. (2011). Determining which mechanisms lead to activation in the motor cortex: a modeling study of transcranial magnetic stimulation using realistic stimulus waveforms and sulcal geometry. Clin. Neurophysiol. 122, 748-758. doi: 10.1016/J.CLINPH.2010.09. 022

Seo, H., and Jun, S. C. (2019). Relation between the electric field and activation of cortical neurons in transcranial electrical stimulation. Brain Stimul. 12, 275-289. doi: 10.1016/j.brs.2018.11.004

Song, X., Zhao, X., Li, X., Liu, S., and Ming, D. (2020). Multi-channel transcranial temporally interfering stimulation (TTIS): application to living mice brain. J. Neural Eng. 18:036003. doi: 10.1088/1741-2552/abd2c9

Sweeney, J. D., Mortimer, J. T., and Durand, D. (1987). "Modeling of Mammalian Myelinated Nerve for Functional Neuromuscular Electrostimulation," in Proceedings of the IEEE 97th Annual Conf. Engineering in Medicine Biology Society, Vol. 9, Boston, MA, 1577-1578.

Vossen, A., Gross, J., and Thut, G. (2015). Alpha power increase after transcranial alternating current stimulation at alpha frequency (a-TACS) reflects plastic changes rather than entrainment. Brain Stimul. 8, 499-508. doi: 10.1016/j.brs. 2014.12.004

Wang, Q., Ding, S. L., Li, Y., Royall, J., Feng, D., Lesnar, P., et al. (2020). The allen mouse brain common coordinate framework: a 3D reference atlas. Cell 181, 936-953.e20. doi: 10.1016/j.cell.2020.04.007

Weber, M. J., Messing, S. B., Rao, H., Detre, J. A., and Thompson-Schill, S. L. (2014). Prefrontal transcranial direct current stimulation alters activation and connectivity in cortical and subcortical reward systems: a TDCS-FMRI study. Hum. Brain Mapp. 35, 3673-3686. doi: 10.1002/hbm.22429

Wongsarnpigoon, A., and Grill, W. M. (2008). Computational modeling of epidural cortical stimulation. J. Neural Eng. 5, 443-454. doi: 10.1088/1741$2560 / 5 / 4 / 009$

Conflict of Interest: The authors declare that the research was conducted in the absence of any commercial or financial relationships that could be construed as a potential conflict of interest.

Copyright (c) 2021 Gomez-Tames, Asai and Hirata. This is an open-access article distributed under the terms of the Creative Commons Attribution License (CC BY). The use, distribution or reproduction in other forums is permitted, provided the original author(s) and the copyright owner(s) are credited and that the original publication in this journal is cited, in accordance with accepted academic practice. No use, distribution or reproduction is permitted which does not comply with these terms. 